\title{
Evolution of Femtosecond Laser Induced Surface Structures at Low Number of Pulses near the Ablation Threshold
}

\author{
Olga VARLAMOVA*1,2 ${ }^{1,}$ Juergen REIF ${ }^{* 1,2}$, \\ *1- Brandenburg. Tech. Univ. BTU Cottbus, Cottbus, Germany \\ Platz der Deutschen Einheit 1, 03046 Cottbus, Germany \\ Email:reif@tu-cottbus.de \\ *2- JointLab Cottbus, Cottbus, Germany
}

\begin{abstract}
We present new features in femtosecond LIPSS on Silicon and $\mathrm{CaF}_{2}$, obtained after a low number of pulses near the ablation threshold. In contrast to the general case of multi-pulse LIPSS, where a strong dependence of the structures on the laser polarization is observed, single-shot exposition of silicon at about the ablation threshold results in a concentric pattern of very regular sub-wavelength ripples following the oval shape of the irradiated spot, without any reference to the laser polarization. Further, a low contrast $( \pm 5 \mathrm{~nm}$ ) center (at highest irradiation density of the Gaussian beam profile) is half surrounded by a high contrast $( \pm 20 \mathrm{~nm})$ region towards the edge - without any change in ripples periodicity. In the high contrast region several perfectly round conical holes are observed, about $50 \mathrm{~nm}$ deep and in diameter comparable to the ripples spacing. These holes are surrounded by a ridge about $20 \mathrm{~nm}$ high. For about 100 pulses well below the ablation threshold on $\mathrm{CaF}_{2}$, no ripples structure can be produced, yet. Nevertheless, the irradiated spot is clearly visible in the SEM picture by a change of SEM contrast. All over and even around the spot, spherical bumps are visible with a rather narrow size-distribution of about $2 \mu \mathrm{m}$ diameter. They almost appear like re-condensed nanoparticles which is, however, not in agreement with the negligible amount of ablated material (ablation depth $<1 \mathrm{~nm}$ ). At higher pulse numbers $(\approx 2,000)$, these bumps persist in the outer range of the ablated spot, whereas in the central region, ripples occur.
\end{abstract}

DOI: $10.2961 /$ jlmn.2013.03.0019

Keywords: LIPSS/ripples; self-organized structure formation; silicon; calcium fluoride; low-dose femtosecond ablation;

\section{Introduction}

The physics of regular surface structures formation ("ripples", LIPSS) upon ultra-fast laser ablation is still greatly disputed. In principle, there are mainly two different scenarios under discussion (Fig. 1): (a) static, quasilithographic ablation from a passive target, reproducing 1by-1 a modulated energy input [1], and (b) a dynamic, active target response via self-organization from a laserproduced (surface) instability [2].

(a) "STATIC" (lithographic) model modulated energy input $=>$ modulated ablation

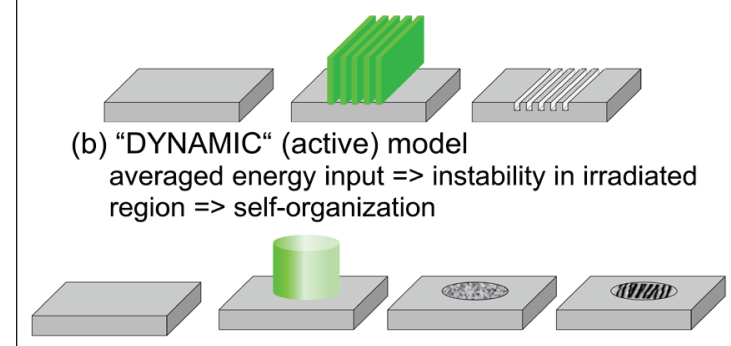

Fig. 1: Main scenarios for the formation of laser-induced periodic surface structures

Though the static, lithographic model has been substantially modified and improved during the recent years, e.g. by considering the role of surface plasmon-polaritons at high electron densities $[3,4,5]$ to account for the observa- tion of sub-wavelength LIPSS, it still lacks from the fact, that the large variety of feature sizes (HSFL [high spatial frequency], LSFL [low spatial frequency LIPSS], and more complex structures like cones etc.) requires all DIFFERENT interactions. In contrast, the dynamic model can account for all such features in ONE, unified approach.

In order to shed more light on the phenomena, we investigate in the present contribution the evolution of structure formation in dependence on the irradiation dose and compare it with the unified approach of our model.

\section{Self-organization model}

Before we present our experimental data, we will first shortly review our dynamic model for the structure formation. Because of the apparent similarities of femtosecond LIPSS with structures produced in ion beam sputtering [6], we adopted an approach which has been very popular in that field. Considering the excited, excited target surface in a similar way as a corrugated thin liquid film which is roughened by material removal, we can describe the irradiation-dose dependent evolution of corrugation height $h$ [7]:

$$
\frac{\partial}{\partial t} h(x, y, t)=-v(\varphi, \theta) \sqrt{1+(\nabla h)^{2}}
$$

where the ablation velocity $v$ depends on the laser fluence.

Taking, further into account that, at the same time, the 
surface is smoothed by surface tension and diffusion, the system becomes instable because of the competing processes, and the system will evolve non-linearly with increasing excitation [8]. In this case, the corrugation evolution can be described by an equation of the Kuramoto-Sivashinsky type $[9,8]$ :

$$
\frac{\partial h}{\partial t}=v_{0}+v \Delta h+\lambda(\nabla h)^{2}-K \Delta^{2} h+\eta,
$$

where $v_{0}$ is the ablation velocity; the second term accounts for the linear dependence of the ablation rate on the surface corrugation (curvature); the third term describes non-linear contributions to erosion at increasing corrugation (slope dependence); the fourth term denotes the loss of corrugation depth by diffusion, and the last term indicates initial surface roughness.

For a comparison of this model with experiments on pulsed laser ablation we must keep in mind that, experimentally, the time evolution has to be translated into a dependence on the number of laser pulses or, more generally, on the irradiation dose.

\subsection{Linear regime}

To analyze the evolution, first the linear regime has to be considered, described by $v \Delta h$, the roughness $\eta$, and the diffusion $K \Delta^{2} h$, where the variation of the surface curvature $\Delta h$ is still negligible. This corresponds to the experimental situation where the irradiated dose is still sufficiently small to avoid substantial crater formation.

Under this condition, we can easily derive the ripples wavelength, $\Lambda$, depending not on the laser wavelength but only on the diffusion rate, $K$, and the parameter for linear erosion, $v$.

$$
\Lambda=2 \pi \sqrt{\frac{2 K}{|v|}} .
$$

Describing the surface excitation, though nor in thermal equilibrium, by a temperature $T$, proportional to the absorbed laser fluence, $F: \quad k T=a F$, the diffusion rate follows an Arrhenius law with activation energy $E_{a}$, corresponding to the binding energy of surface atoms:

$$
K(T) \propto \frac{1}{k T} \exp \left\{\frac{E_{a}}{k T}\right\} .
$$

Since the erosion parameter $v=b F$ also depends linearly on the fluence ( $a, b$ are constants of proportionality), the ripples wavelength can be expressed by:

$$
\Lambda=2 \pi \sqrt{\frac{2 K}{|v|}}=\sqrt{\frac{2 / a F^{\exp }\left\{-E_{a} / a F\right\}}{b F}} \propto \frac{1}{F} \exp \left\{-\frac{E_{a}}{2 a F}\right\}
$$

This behavior is shown, generally", in Fig. 2, where we can distinguish three regimes, depending whether the de-

\footnotetext{
* A quantitative determination would require the knowledge of the detailed magnitude of all parameters $(K, a, v \ldots)$
}

posited energy is smaller, about equal, or larger than the surface binding energy. These regimes are typical for dielectric, semiconductor, or metallic targets, correspondingly.

As can be seen in the Figure, for dielectric targets, the LIPSS period increases with increasing dose, a coarsening of the structures must be observed. On the other hand, for metals the LIPSS wavelength decreases, and the structures become finer. For semiconductors, like silicon, the feature width in the linear regime should not vary too much.

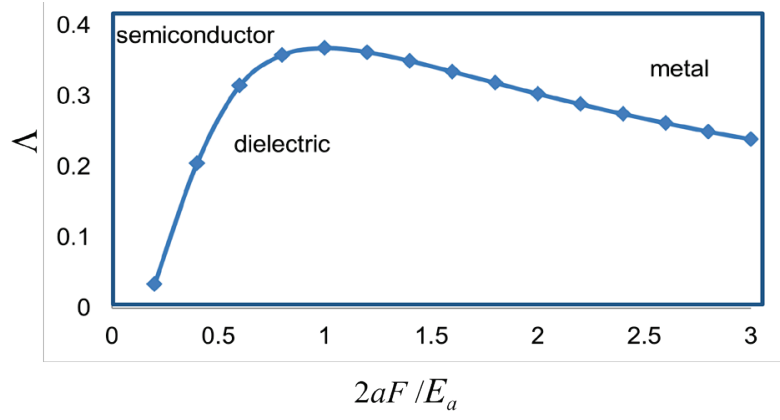

Fig. 2. Linear regime: dependence of ripples wavelength/spacing $\Lambda$ (dimensionless parameter) on incident fluence $F$ (cf. Eq. (5))

\subsection{Non-linear regime}

Upon further increasing the irradiation dose (i.e. evolution time in Eq. (2)), the surface corrugation becomes more and more pronounced, and the nonlinear coupling terms in Eq. (2) gain importance. This affects both, the diffusion (surface smoothing) and the erosion (surface roughening). As a consequence, structures will start to coalesce, resulting in bifurcations, broad interconnects and, finally, less regular, large agglomerates.

\section{Experimental results and discussion}

In our experiments to investigate the early phase of structure formation, we investigated the influence of increasing dose (i.e. increasing number of accumulated pulses) of an ultra-short-pulse laser (100 fs, $800 \mathrm{~nm}$, linearly polarized, focused to a spot of about $5 \times 10^{-5} \mathrm{~cm}^{2}$ ) for two different scenarios: (a) we irradiated dielectric $\mathrm{CaF}_{2}$ at a very low level of $8.2 \mathrm{TW} / \mathrm{cm}^{2}$ (i.e. $25 \%$ of the ablation threshold) with 100 resp. 10,000 pulses, in both cases staying well within the linear regime. (b) In the second scenario, we irradiated silicon at a fluence of $215 \mathrm{~mJ} / \mathrm{cm}^{2}$ (i.e., just at the ablation threshold). Here, we considered the effect of a single pulse and then increased the number of accumulated pulses from 10 pulses (linear regime) over 50 pulses (onset of non-linear regime) to 1,000 pulses (crater formation).

\subsection{Linear regime \\ $3.1 .1 \mathrm{CaF}_{2}, 25 \%$ of threshold}

At very low dose (100 pulses), no regular structures were developed. However, the electron reflectivity in the irradiated spot was increased, as can be seen in light-grey area in the SEM image of Fig. 3a). In addition, a large number of (semi-spherical) bumps, swollen above the pristine surface level) occurred, with diameters of a few $\mu \mathrm{m}$ and distributed over a large area, even beyond the visible spot. Details of one such bump are given in the AFM image 
of a restricted area in the spot (Fig. 3b) and a cross-section (Fig. 3c). Even though, at first sight, these bumps might appear as re-deposited material from anterior ablation (cf. "grey" surface modification) the total volume of all bumps, as estimated from AFM scan, should be much larger than the ablated volume, estimated from spot depth and area. This strongly supports the idea of laser induced material swelling. a)

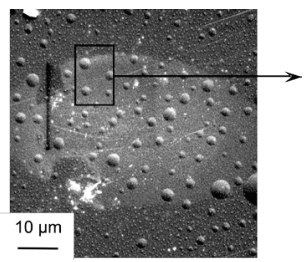

b)

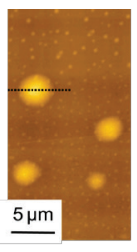

c)

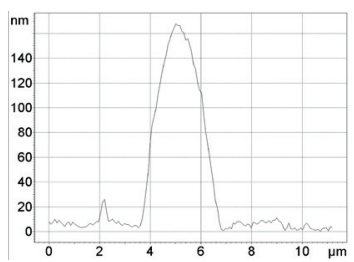

Fig. 3. Irradiated area morphology on $\mathrm{CaF}_{2}$, subjected to 100 pulses @ 25\% of ablation threshold;

When increasing the dose (number of pulses) by a factor of 100, the resulting much larger number of such bumps or blisters starts to agglomerate and form long, parallel arrays (Fig. 4).

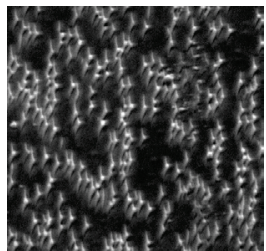

Fig. 4. Detail of morphology on $\mathrm{CaF}_{2}$, subjected to 10,000 pulses (a) $25 \%$ of ablation threshold

\subsubsection{Silicon, about threshold $\left(215 \mathrm{~mJ} / \mathrm{cm}^{2}\right)$}

For irradiation of silicon at about the ablation threshold, LIPSS can be observed already after a single shot (Fig. 5). However, the orientation follows the spot while the influence of the laser polarization (double arrow) is not the predominant parameter.

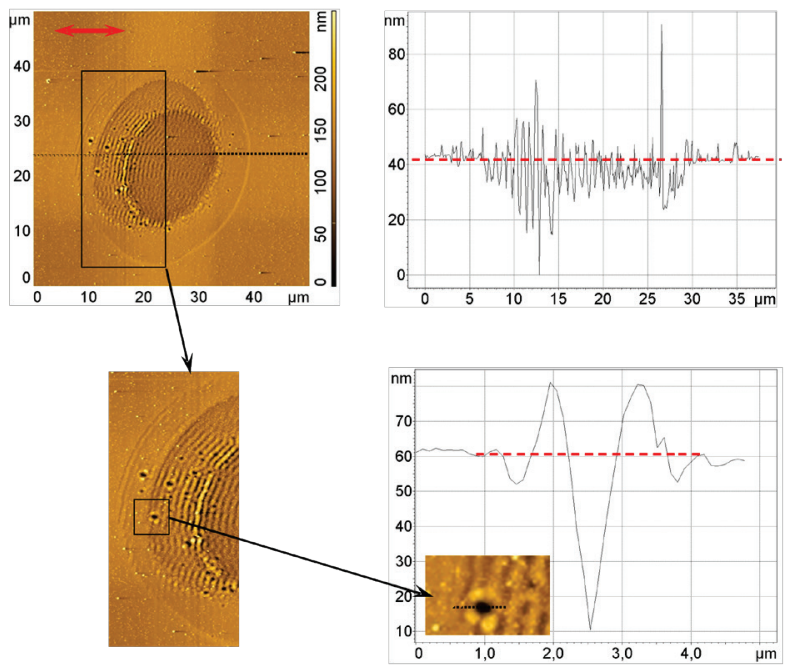

Fig. 5. LIPSS on silicon after a single shot at about threshold. The double arrow in the upper left panel indicates the laser polarization. Lower panels: magnified details of the spot (cf. frames)
The ripples structure with a period of about $600 \mathrm{~nm}$ oscillates with amplitude of up to $25 \mathrm{~nm}$ above and below the pristine surface (dashed line). The origin of the left-right asymmetry is not yet clear. It seems not to be related to the slightly inclined incidence, indicated by the oval spot shape. Note the circular holes in the rippled area, with a diameter of about the ripples wavelength (lower details in Fig. 5). As can be seen from the AFM line-trace (right), they about three times as deep as the ripples valleys and are surrounded by a ridge of about the ripples crests height.

After 10 pulses, the LIPSS are already fully developed (Fig. 6). Though this is still the linear regime, the dose is already so high that the ripples wavelength is about $750 \mathrm{~nm}$ (cf. Fig. 2). The ripples direction is now mainly perpendicular to the laser polarization. However, the holes, already observed for single-pulse irradiation, occur now as equivalent centers of radial ripples arrays. Further, a modulation in SEM contrast can be seen perpendicular to the ripples orientation.

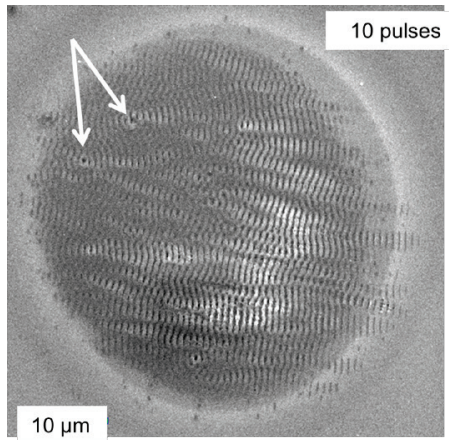

Fig. 6. LIPSS pattern on silicon after 10 pulses @ $215 \mathrm{~mJ} / \mathrm{cm}^{2}$ (about ablation threshold). The polarization is nearly horizontal. The arrows point to two remnant holes (cf. Fig. 5), acting as centers for radial ripples arrays.

\subsection{Nonlinear regime of pattern formation}

When further increasing the dose by a factor of 5 , the transition to the non-linear regime sets in. A significantly coarser horizontal structure is observed, perpendicular to the lower dose one. As is shown in the magnified detail of the central spot area (magnified detail in Fig. 7), these structures are interlinked by a system of fine vertical ladder spokes.

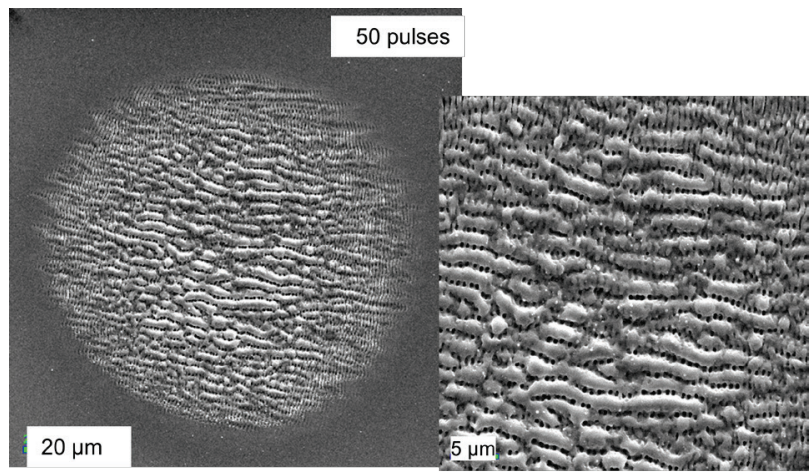

Fig. 7. Transition to the non-linear regime of self-organized pattern formation: coalescence, coarsening.

Right panel: magnified detail of the spot center. 
A comparison between details of the 10-pulse (linear regime) and 50-pulse (beginning non-linear regime) morphologies, magnified to the same scale, reveals a strong correlation between the two sets of structures (Fig. 8):

Evidently, the fine ladder spokes interlinking the coarse structures (50 pulses) are remnants of the fine ripples of the linear regime. On the other hand, the transverse modulations of the low-dose LIPSS coalesce into the broad vertical ripples in the non-linear regime. This becomes visible by the partial superposition of the two patterns in Fig. 8 .

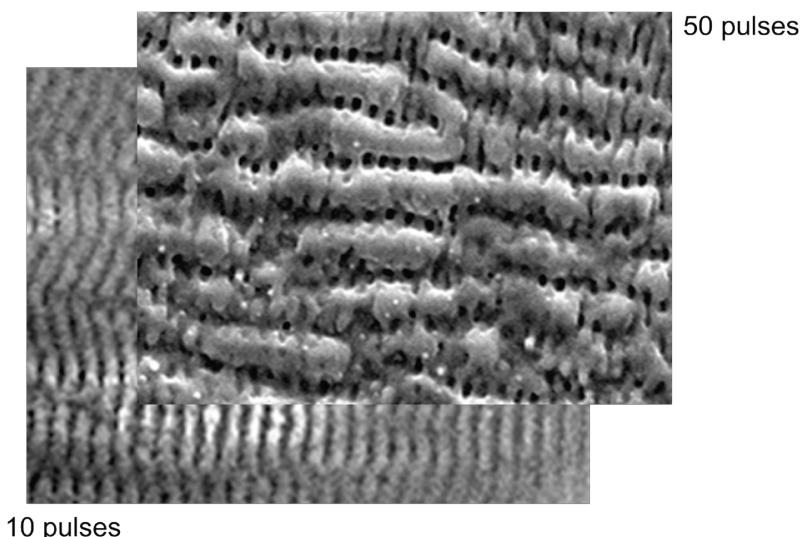

Fig. 8: Comparison of morphology details obtained at 10 pulses (left) and 50 pulses (right). The details from Figs. 6, 7 are magnified to the same scale.

At very high irradiation dose (1,000 pulses), a deep ablation crater has developed (Fig. 9, right panel). But still LIPSS are observable both at the inner crater walls and - as a ring - in the region of the foot of the beam profile. Obviously, in the strongly irradiated area the structure coarsening has further increased, including cones and pillars. At the very edge, however, still fine ripples are visible, like in Fig. 6.
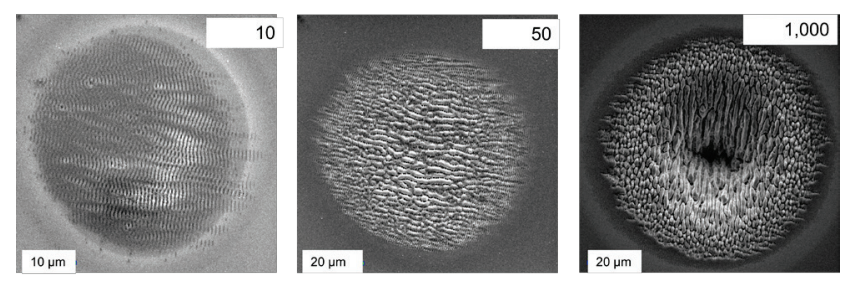

Fig. 9: Evolution of irradiated spot morphology with increasing dose at threshold fluence

\section{Conclusion}

We have shown that there is a continuous evolution of the LIPSS formation, depending on the irradiation dose. Starting from precursor states with unordered bumps and swellings, these bumps agglomerate into longer, ordered arrays. It follows a regular surface corrugation, oscillating below and above the pristine surface level. Then, in a linear growth regime, long regular ripples develop, becoming coarser with increased dose for non-metals. Only in this phase, the important role of the laser polarization for the ripples alignment sets in. At still higher dose, the material response turns nonlinear, and more complex structures develop, involving coalescence from previously generated patterns. All this evolution is well described by our selforganization model in Eq. (2) [2], indicating that all LIPSS formation is driven by ONE underlying physical phenomenon. This is in contrast to the lithographic model which requires the energy absorption distribution to vary for all different types of structure.

\section{Acknowledgments}

We gratefully acknowledge fruitful discussions with Tzanimir Arguirov, Jürgen Bertram, Mourad Bounhalli, Marco Muth, Markus Ratzke, Florenta Costache, Martin Schade, and Hartmut Leipner

\section{References}

[1] J.F. Young, J.S Preston, H.M. van Driel, J.E. Sipe, Phys. Rev. B 27, 1155 (1983)

[2] J. Reif, O. Varlamova, S. Varlamov, M. Bestehorn, Appl. Phys A 104, 969 - 973 (2011)

[3] J. Bonse, A.Rosenfeld, J.Krüger, J. Appl. Phys. 106, 104910 (2009)

[4] F. Garrelie, J. P. Colombier, F. Pigeon, S. Tonchev, N. Faure, M. Bounhalli, S. Reynaud, and O. Parriaux, Opt. Express 19, 9035 (2011)

[5] G. Miyaji and K. Miyazaki, Opt. Express 16, 16265 (2008)

[6] See, e.g., B. Ziberi, F. Frost, Th. Höche, B. Rauschenbach, Phys. Rev. B 72, 235310 (2005)

[7] P. Sigmund, J. Mat. Sci. 8, 1545 (1973)

[8] R. Cuerno, A.-L. Barabási, Phys. Rev. Lett. 74, 4746 (1995)

[9] Y. Kuramoto, T. Tsuzuki, Progr. Theor. Phys. 55, 356 (1976)

G. Sivashinsky, Acta Astronautica 4,1177 (1977)

(Received: October 2, 2013, Accepted: December 12, 2013) 